Schulze: Elem. Zusammensetz. d. Weizenstärke etc. 311

\title{
Die elementare Zusammensetznng der Weizenstärke und die Einwirkung von verdünnter Essigsäure auf Stärkemehl;
}

\author{
von \\ Ludwig Schulze.
}

\section{Die elementare Zusammensetzung der Weizenstärke.}

Das interessante chemische Verhalten der Stärke, ihre grosse Bedeutung in dem Lebensprocesse der Pflanzen, hat schon frühzeitig die Chemiker veranlasst, dieselbe in den Kreis ihrer Untersnchungen zu ziehen. Nächst den Umwandlungsproducten, welche durch Behandeln der Stärke mit verschiedenen chemischen Reagentien, z. B. verdünnten Mineralsäuren, entstehen, war es besonders die Frage über die chemische Zusammensetzung der Stärke, mit welcher sich die Chemiker lange Zeit hindurch beschäftigten. Die früher allgemein angenommene Formel $\mathrm{C}_{6} \mathrm{H}_{10} \mathrm{O}_{5}$ wurde zuerst von Naegeli angezweifelt und von diesem auf Grund verschiedener Elementaranalysen die Formel $\mathrm{C}_{36} \mathrm{H}_{62} \mathrm{O}_{31}$ als diejenige vorgeschlagen, welche den bekannten Erfahrungen am besten entspräche.

Später versuchte Sachsse durch Bestimmung des aus der Stärke mittelst verdünnter Säuren gebildeten Traubenzuckers dieselbe Frage zu entscheiden, und auch er kam zu dem Schlusse, dass die von $\mathrm{Naegeli}$ vorgeschlagene Formel $\mathrm{C}_{36} \mathrm{H}_{62} \mathrm{O}_{31}$ sich den Thatsachen am besten anschliesse. Die schwebende Frage wäre hierdurch zum endgültigen Abschluss gebracht, wenn nicht in neuester Zeit durch die schönen und umfassenden Untersuchungen von Soxhlet klar gelegt wäre, dass die bisherigen Methoden der Bestimmung des Traubenzuckers grosse Mängel enthielten, und da Sachsse's Beweissführung lediglich auf der von ihm selbst aufgefundenen Bestimmung des Traubenzuckers mittelst Cyanquecksilbers beruht, so erschien es geboten, nochmals eine sorgfältige Prüfung der vorliegenden Frage vorzunehmen. 


\section{Schulze: Die elementare Zusammensetzung}

Durch in neuester Zeit angestellte Beobachtungen über das spec. Drehungsvermögen des Traubenzuckers, über das spec. Gewicht desselben und endlich über das Verhalten des Traubenzuckers gegen alkalische Kupferlösung durch Soxhlet, Allihn, Tollens, Salomon u. A. sind uns neue Mittel an die Hand gegeben, die bisher noch zweifelhafte Frage über die chemische Zusammensetzung der Stärke ihrer Entscheidung entgegenzuführen.

Da meine Untersuchungen, durch welche ich den sicheren Beweis zu führen glaube, dass der Weizenstärke die ihr bislang zugeschriebene Zusammensetzung $\mathrm{C}_{6} \mathrm{H}_{10} \mathrm{O}_{5}$ und nicht die von $\mathrm{Na}$ egeli vorgeschlagene $\mathrm{C}_{36} \mathrm{H}_{62} \mathrm{O}_{31}$ zukommt, lediglich auf den Beobachtungen dieser Forscher beruhen, so wird mir im Verlauf dieser Arbeit noch verschiedentlich Gelegenheit geboten sein, auf die Wichtigkeit derselben hinzuweisen. Die Stärke, die zu meinen sämmtlichen Versuchen, soweit diese über die Zusammensetzung derselben in Frage kommen, benutzt wurde, war Weizenstärke, welche durch Behandeln mit einer verdünnten Lösung von Natronhydrat, einer einprocentigen Salzsäure und nachherigem Auswaschen mit Wasser bis zum Verschwinden der letzten Spur von Säure gereinigt war. - Die Reinigung der Stärke, speciell der Weizenstärke, welche zu wissenschaftlichen Zwecken verwandt werden soll nach der eben beschriebenen Methode, ist deshalb erforderlich, weil den im Handel rorkommenden Producten mehr oder weniger mineralische Substanzen und fremde Körper organischer Natur beigemengt sind.

Bevor ich zur näheren Beschreibung der von mir ausgeführten Versuche selbst übergehe, sei es mir gestattet, erst noch die Analyse der von mir verwandten Stärke hier anzufïhren.

Analyse der Weizenstärke.

Da die Analyse der Weizenstärke nach denselben Vorschriften, welche Dr. Salomon in diesem Bande des Journals (S. $84 \mathrm{ff}$ ) für die Analyse der Stärke gegeben hat, ausgeführt wurde, so kann ich mithin von einer näheren Beschreibung absehen und mich lediglich auf die Angaben der erhaltenen Zahlenwerthe beschränken. 
Wasserbestimmung.

Probe I. 5,463 Grm. Stärke wurden abgewogen und in einem mit doppelten Wandungen und Thermoregulator versehenen Luftbade (der zwischen beiden Wandungen befindliche Raum war mit Oel gefüllt) bei Temperaturen von $100^{\circ}$ bis $130^{\circ}$ getrocknet.

Das Trocknen bei ein und derselben Temperatur wurde selbstverständlich so lange fortgesetzt, als noch eine Gewichtsabnahme erfolgte.

Die so getrockneten 5,463 Grm. Stärke gaben:

$$
\begin{aligned}
\text { bei } 100^{\circ} & =4,392 & \text { Grm. Trockensubstanz } \\
" 110^{\circ} & =4,385 \quad " & \\
" 120^{\circ} & =4,366 \quad " & \\
" 130^{\circ} & =4,347 \quad " &
\end{aligned}
$$

Probe II. 6,263 Grm. Stärke wurden unter denselben Bedingungen, wie Probe I getrocknet und hinterliessen:

$$
\begin{aligned}
\text { bei } 100^{\circ} & =5,038 & \text { Grm. Trockensubstanz } \\
\prime 110^{\circ} & =5,026 \quad " & \\
" 1 & 120^{\circ}=5,001 \quad " & \\
" 130^{\circ} & =4,99 \quad " &
\end{aligned}
$$

Probe III. 5,709 Grm. Stärke wurden unter denselben Bedingungen, wie Probe I getrocknet und hinterliessen:

$$
\begin{array}{rlrl}
\text { bei } 100^{\circ} & =4,588 & \text { Grm. Trockensubstanz } \\
" 110^{\circ} & =4,573 \quad " & \\
" 1 & 120^{\circ}=4,556 \quad " & " \\
" 130^{\circ} & =4,542 \quad " &
\end{array}
$$

Aus den eben angeführten Zahlenwerthen ergiebt sich bei $120^{\circ}$ ein Wassergehalt von $20,143 \%$.

Der besseren Uebersicht halber werde ich in der nachfolgenden Tabelle den Wassergehalt bei den verschiedenen Temperaturen in Procenten angeben.

\begin{tabular}{c|c|c|c|c|c|c|c}
\hline $\begin{array}{c}\text { Num. } \\
\text { des }\end{array}$ & $\begin{array}{c}\text { bei } \\
\text { Vers. }\end{array}$ & $\begin{array}{c}\text { Differenz } \\
\text { bei } \\
100-110^{\circ}\end{array}$ & $\begin{array}{c}\text { bei } \\
110^{\circ}\end{array}$ & $\begin{array}{c}\text { Differenz } \\
\text { bei } \\
110-120^{\circ}\end{array}$ & $\begin{array}{c}\text { bei } \\
120^{\circ}\end{array}$ & $\begin{array}{c}\text { Differenz } \\
\text { bei } \\
120-130^{\circ}\end{array}$ & $\begin{array}{c}\text { bei } \\
130^{\circ}\end{array}$ \\
\hline \hline I. & $\mathbf{1 9 , 6 0 4}$ & 0,129 & 19,733 & 0,347 & 20,08 & 0,348 & 20,428 \\
II. & 19,559 & 0,191 & 19,75 & 0,400 & 20,15 & 0,175 & 20,325 \\
III. & 19,635 & 0,263 & 19,898 & 0,298 & $\mathbf{2 0 , 1 9 6}$ & 0,245 & $\mathbf{2 0 , 4 4 1}$
\end{tabular}




\section{Schulze: Die elementare Zusammensetzung}

Aschenbestimmung.

Aus gleich anzuführenden Zahlwerthen ergiebt sich für meine Weizenstärke ein mittlerer Aschengehalt von $0,061 \%$.

Probe I. 26,832 Grm. lufttrockener Stärke $=22,335 \mathrm{Grm}$. wasserfreier hinterliessen $0,014 \mathrm{Grm}$. Asche gleich $0,032 \%$.

Probe II. 21,846 Grm. lufttrockener Stärke = 18,184 Grm. wasserfreier hinterliessen $0,011 \mathrm{Grm}$. Asche gleich $0,06 \%$.

Bestimmung des in Säuren unlöslichen Rückstandes.

Probe I. 3,604 Grm. Iufttrockene Stärke $=3$ Grm. wasscrfreier gaben $0,03 \mathrm{Grm} .=1 \%$ Rückstand.

Probe II. 3,604 Grm. lufttrockener Stärke $=3 \mathrm{Grm}$. wasserfreier gaben $0,036 \mathrm{Grm} .=1,2^{0}{ }_{0}$ Rückstand. Im Mittel demnach $1,1 \%$ unlöslicher Rückstand.

Aus den eben angeführten Resultaten ergiebt sich, dass die von mir in den weiteren Versuchen angewandte Weizenstärke folgende Zusammensetzung besitzt:

$\begin{array}{lr}\text { Wasser } & 20,143 \\ \text { Asche } & 0,061 \\ \text { Unlösl. Rückstand } & 1,1 \\ \text { Reine Stärke } \quad & 78,696 \\ & 100,000\end{array}$

Hieraus berechnet sich nun die $100 \mathrm{Grm}$. absolut reiner Stärke entsprechende Menge lufttrockenerStärkezu 127,07Grm.

Von einer Bestimmung des durch Salzsäure gebildeten Traubenzuckers habe ich bei der Analyse Abstand genommen, weil auf derselben meine erste Versuchsreihe zur Feststellung der Stärkeformel basirt und aus den hierbei erhaltenen Resultaten mit Leichtigkeit die reine Stärke berechnet werden kann.

\section{Versuche zur Feststellung der Stärkeformel.}

Bei den Untersuchungen, welche ich nun angestellt habe, um einen endgültigen Abschluss über die Formel der Weizenstärke ${ }^{1}$ ) zu erzielen, habe ich sowohl das chemische

1) Dr. Salomon, welcher nich zur Ausführung dieser Arbeit veranlasste, hat dieselbe Frage in Betreff der Reiss- und Kartoffelstärke entschieden. (Dies. Journ. [2] 26, 324 и. \$2 348 .) 
Verhalten der Stärke gegen verdünnte Säuren als auch ihre elementare Zusammensetzung möglichst genau geprüft und die Resultate im Nachfolgenden niedergelegt. Um aber bei der Bestimmung des durch die Behandlung der Stärke mit verdünnten Säuren entstehenden Zuckers nicht von einer einzigen Methode abhängig zu sein, habe ich mich nicht damit begnügt, die Menge desselben durch Feststellung des Reductionswerthes allein zu erfahren, sondern es ist, wie aus den unten angegebenen Daten hervorgeht, sowohl das spec. Gewicht, als auch die optische Wirkung des Traubenzuckers zu Hülfe genommen, und glaube ich, so in jeder Hinsicht sichere Grundlagen zur Lösung der schwebenden Frage erhalten zu haben.

A. Durch Verzuckerung mittelst Salzäure und Bestimmung des Traubenzuckers nach Allihn's Verfabren.

Fast sämmtliche Forscher, welche sich bisher mit der quantitativen Ueberführung der Stärke in Traubenzucker beschäftigten, bedienten sich zur Inversion derselben der verdünnten Schwefelsäure und erhielten auf diese Weise aus 100 Thln. Stärke 107--110 Thle., während nach der Theorie 111,11 Thle. Traubenzucker entstehen sollen.

Da es nun bei meinen Versuchen ausschliesslich darauf ankam, eine möglichst vollkommene Verzuckerung der Stärke herbeizuführen, diese aber nach den bisherigen Vorschriften mit Schwefelsäure nicht zu erwarten war, so war ich gezwungen, mich nach einem anderen Verfahren umzusehen, und fand ich in der von Sachsse (derselbe bedient sich zur Hydratisirung der Stärke der Salzsäure, Chem. Centralbl. 1877, S. 732) empfohlenen Methode zur Inversion der Stärke ein Mittel, welches mir die erwünschten Resultate lieferte.

Die Bestimmung des erhaltenen Traubenzuckers geschieht am zweckmässigsten nach Allihn's ${ }^{1}$ ) gravimetrischer Methode.

Bei den Versuchen, welche Herr Dr. Salomon und

1) Dies. Journ. [2] 22, S. 46. 


\section{Schulze: Die elementare Zusammensetzung}

ich über die Verwendbarkeit dieser Methode anstellten, erhielten wir bei Anwendung von reinen Traubenzuckerlösungen, auch wenn dieselben nicht die von Allihn vorgeschriebene Concentration (nämlich $1 \%$ ) besassen, gute Resultate, und erwies sich dadurch die Methode als durchaus zuverlässig. Dasselbe bestätigte sich durch Versuche, welche Herr Dr. Salomon mit Kartoffel- und Reisstärke ausführte. Als ich jedoch diese Erfahrungen auf die Weizenstärke anwandte, stiess ich auf Hindernisse, indem nämlich Zahlen erhalten wurden, welche den berechneten Werth durchschnittlich um $2-3^{0} / 0$ überstiegen. Die Zahlenwerthe wurden aber dem theoretischen Werthe entsprechend, als ich annähernd einprocentige Lösungen anwandte und sumit fas die ganze in Anwendung kommende Kupferlösung reducirtet demnach also eine Nachreduction, wie Allihn diese Erscheinung nennt, nicht eintreten konnte. $\mathrm{Ob}$ übrigens in der sogenannten Nachreduction der Grund zu suchen ist, dass ich zu hohe Zahlenwerthe erhielt, oder ob derselbe darin zu suchen ist, dass in der Weizenstärke ein Körper vorhanden oder bei der Inversion derselben entsteht, welcher alkalische Kupferlösung ebenfalls reducirt, will ich dahingestellt sein lassen. Für die letztere Annahme spricht der Grund, dass sowohl bei der Kartoffel- als auch bei der Reisstärke diese Erscheinung nicht beobachtet wurde. Sachsse hat übrigens seiner Zeit bei der Weizenstärke dieselbe Erscheinung wahrgenommen.

Es ergiebt sich hieraus, dass man bei Anwendung der Allihn'schen Methode, wenn man nicht mit ganz reinen Traubenzuckerlösungen operirt, streng darauf zu achten hat, dass dieselben annähernd einen Gehalt von $1 \%$ besitzen.

Im Folgenden sind die Versuche mitgetheilt, welche ich über die Verzuckerung der Weizenstärke anstellte und die beweisen sollen, dass der Stärke die ihr bislang zugeschriebene Formel $\mathrm{C}_{6} \mathrm{H}_{10} \mathrm{O}_{5}$ zukommt. Die Versuche sind unter Berücksichtigung der oben angeführten Vorsichtsmassregeln ausgeführt.

Versuch I. 3,458 Grm. lufttrockener Stärke (entsprechend 2,721 Grm. absolut reiner Stärke) wurden mit $20 \mathrm{Ccm}$. Salzsäure vom 
spec. Gewwicht 1,125 und $180 \mathrm{Ccm}$. Wasser 3 Stunden lang am Rückflusskühler gekocht, darauf zu $250 \mathrm{Ccm}$. aufgefïllt und mit dieser Lösung die Zuckerbestimmungen ausgeführt. Zu jeder Zuckerbestimmung wurden 3 Versuche angestellt und ron diesen dann das arithmetische Mittel genommen.

Da die jetzt folgenden Versuche alle eine stärkere Concentration als $1 \%$ besassen, so wurden zur Reduction nur $20 \mathrm{Ccm}$. verwandt.

20 Ccm. gaben im Mittel 0,448 Grm. Cu=0,241 Grm. Zucker, in $250 \mathrm{Ccm}$. demnach $0,241 \times 12,5=3,0125 \mathrm{Grm}$. Zucker.

Hieraus berechnet sich ein Zuckergehalt von 110,712 p. c.

Versuch II. 3,1905 Grm. lufttrockener Stärke (gleich 2,511 Grm. absolut reiner Stärke) sind in derselben Weise wie Versuch I behandelt.

$20 \mathrm{Ccm}$. Lösung gaben $0,4205 \mathrm{Grm}$. $\mathrm{Cu}=0,2248 \mathrm{Grm}$. Zucker, in $250 \mathrm{Ccm}$. demnach $0,2248 \times 12,5=2,81$ Grm. Zncker=111,907 p. c.

Versuch III. 3,547 Grm. lufttrockener Stärke (entsprechend 2,791 Grm. absolut reiner Stärke) sind in derselben Weise wie Versuch I behandelt.

$20 \mathrm{Ccm}$. Lösung gaben 0,4585 Grm. $\mathrm{Cu}=0,2471$ Grm. Zucker, in $250 \mathrm{Ccm}$. Lösung $0,2471 \times 12,5=3,0947$ Grm. Zucker gleich 110,8 p. c.

Versuch IV. 3,311 Grm. lufttrockener Stärke (entsprechend 2,605 Grm. absolut reiner Stärke) sind wie Versuch I behandelt.

20 Ccm. Lösung gaben 0,43 Grm. $\mathrm{Cu}=0,2304$ Grm. Zucker, in $250 \mathrm{Ccm}$. Lösung $0,2304 \times 12,5=2,88$ Grm. Zucker $=110,556$ p. c.

Versuch V. 3,2755 Grm. lufttrockener Stärke (entsprechend 2,577 Grm. absolut reiner Stärke) sind wie Versuch I behandelt.

$20 \mathrm{Ccm}$. Lösung gaben 0,429 Grm. $\mathrm{Cu}=0,2298 \mathrm{Grm}$. Zucker, in 250 Ccm. Lösung $0,2298 \times 12,5=2,872$ Grm. Zucker gleich 111,447 p. c.

Der besseren Uebersicht halber sei es mir gestattet, in einer Tabelle die eben beschriebenen Versuche nochmals. aufzuführen.

\begin{tabular}{|c|c|c|c|c|c|c|}
\hline $\begin{array}{c}\text { Num. } \\
\text { des } \\
\text { Vers. }\end{array}$ & $\begin{array}{l}\text { Angew. } \\
\text { lufttrock. } \\
\text { Stärke. }\end{array}$ & $\begin{array}{c}\text { Entspr. } \\
\text { reine } \\
\text { Stärke. }\end{array}$ & $\begin{array}{l}\text { Reducirt. } \\
\text { Kupfer } \\
\text { in Mgrm. }\end{array}$ & $\begin{array}{l}\text { Entspr. } \\
\text { Traubenz. } \\
\text { in Mgrm. }\end{array}$ & $\begin{array}{l}\text { In } 250 \text { Ccm. } \\
\text { enthalt. } \\
\text { Traubenz. } \\
\text { in Grm. }\end{array}$ & $\begin{array}{l}\text { Procente } \\
\text { Traubenz. }\end{array}$ \\
\hline I. & 3,458 & 2,721 & 448 & 241 & 3,0125 & 110,712 \\
\hline II. & 3,1905 & 2,511 & 420,5 & 224,8 & 2,81 & 111,907 \\
\hline III. & 3,547 & 2,791 & 458,5 & 246,3 & 3,0787 & 110,308 \\
\hline IV. & 3,311 & 2,605 & 430 & 230,4 & 2,88 & 110,556 \\
\hline V. & 3,2755 & 2,577 & 429 & 229,8 & 2,872 & 111,447 \\
\hline
\end{tabular}

Hieraus berechnet sich ein mittlerer Procentgehalt von 110,986, also fast genau der von der Theorie verlangte Werth. Aus den eben angeführten Versuchen lässt sich 


\section{Schulze: Die elementare Zusanmensetzung}

mit Leichtigkeit der Gehalt an absolut reiner Stärke berechnen. Beispielsweise will ich hier den Stärkegehalt von Versuch I berechnen.

Da 111,1 Grm. Traubenzucker $100 \mathrm{Grm}$. absolut reiner Stärke entsprechend sind, so entsprechen die gefundenen 3,0125 Grm. Zucker 2,711 Grm. reiner Stärke.

Aus den eben beschriebenen fün Versuchen scheint mir zur Genüge hervorzugehen, dass die Verzuckerung der Stärke nach der bisher üblichen Formel

$$
\mathrm{C}_{6} \mathrm{H}_{10} \mathrm{O}_{5}+\mathrm{H}_{2} \mathrm{O}=\mathrm{C}_{6} \mathrm{H}_{12} \mathrm{O}_{6}
$$

vor sich geht d. h. aus 162 Theilen absolut reiner Stärke entstehen 180 Theile Traubenzucker, oder, was dasselbe ist. aus 100 Theilen absolut reiner Stärke 111,11 Theile Traubenzucker.

B. Durch Verzuckerung mittelst Salzsäure und Bestimmung des Traubenzuckers durch das spec. Gewicht.

In neuester Zeit hat Dr. Salom on ${ }^{1}$ ) das spec. Gewicht einer 10proc. reinen Traubenzuckerlösung (10 Grm. zu 100 Ccm. gelöst) bei $17,5^{\circ}$ zu 1,0381 ermittelt. Diese Zahl habe ich meinen sämmtlichen Versuchen, welche ich zur Ermittelung des Traubenzuckergehaltes durch das spec. Gewicht anstellte, zu Grunde gelegt.

Angenommen, wir hätten das spec. Gewicht einer Lösung zu 1,042 gefunden; d. h. das Gewicht von $100 \mathrm{Ccm}$. Wasser ist um 4,2 Grm. vergrössert. Da nun aber eine Gewichtszunahme von 3,81 Grm. 10 Grm. Traubenzucker entspricht, so erhalten wir die Proportion:

$3,81: 10=4,2: x ; x=11,023$ Grm. Traubenzucker in $100 \mathrm{Ccm}$. oder in $100 \mathrm{Grm}$. Lösung $1,042: 11,023=10,636$ Grm. Traubenzucker.

$\mathrm{Um}$ jedoch diese Rechnung zu vermeiden, ist von Herrn Dr. Salomon ${ }^{2}$ ) eine Tabelle angefertigt, welche es ermöglicht, aus dem gefundenen spec. Gewicht die in $100 \mathrm{Ccm}$. der Lösung enthaltene Menge Traubenzucker direkt abzulesen.

i) Repert. analyt. Chem. 1881, S. 310 n. dies. Journ. [2] 98, 96.

2) Repert. analyt. Chem. 1s81, S. 310 . 
Ich gehe jetzt zur Beschreibung der einzelnen Versuche selbst über.

Versuch I. $31,767 \mathrm{Grm}$. lufttrockener Stärke (entsprechend $25 \mathrm{Grm}$ absolut reiner Stärke) wurden 3 Stunden lang am Rückflusskühler mit $20 \mathrm{Ccm}$. Salzsäure vom spec. Gewicht 1,125 und $180 \mathrm{Ccm}$. Wasser im Kochsalzbade erhitzt. (Das Kochsalzbad wurde deshalb angewandt, weil sein Siedepunkt um $7^{\circ}$ höher liegt als der des Wassers.) Daranf wurde die Lösung zu $250 \mathrm{Ccm}$. aufgefüllt, und dann in einem genau $55 \mathrm{Ccm}$. enthaltenden Kölbchen das spec. Gewicht bestimmt. Dasselbe ergab sich zu 1,05256, zieht man hiervon das einer 2 proe. Salzäure entsprechende spec. Gewicht, gleich 1,01, ab, so erhält man ein spec. Gewicht von 1,04256 .

Daraus berechnet sich der Traubenzuckergehalt zu: $3,81: 10=$ $4,256: x ; x=11,18 \mathrm{Grm}$. in $100 \mathrm{Ccm}$. Lösung; in $250 \mathrm{Ccm}$. sind demnach 27,950 Grm. Zucker, oder aus $25 \mathrm{Grm}$. reiner Stärke sind 27,95 Grm. Zucker entstanden. In Procenten ausgedruickt 111,8.

Versuch II. Es wurde genau dieselbe Menge Stärke und in derselben Weise wie in Versuch I verzuckert.

Gef. spec. Gew. $=1,05258-1,01=1,04258$, entspricht 11,176 Grim. Zucker in $100 \mathrm{Ccm}$. Aus $25 \mathrm{Grm}$. reiner Stärke sind demnach 27,937 Grm. Zucker entstanden, oder in Procenten 111,76.

Versuch III. Genau in derselben Weise angestellt wie Versuch I.

Gef. spec. Gew. $=1,0524-1,01=1,0424$, entsprechend $11,128 \mathrm{Grm}$. Traubenzucker in $100 \mathrm{Ccm}$. Aus $25 \mathrm{Grm}$. reiner Stärke sind demnach 27,82 Grm. Traubenzucker entstanden, oder in Procenten 111,28.

Versuch IV. Genau in derselben Weise angestellt wie Versuch I.

Gef. spec. Gew. $=1,0522-1,01=1,0422$, entsprechend 11,076 Grm. Zucker in $100 \mathrm{Ccm}$. Aus $25 \mathrm{Grm}$. Stärke sind dernnach 27,69 Grm. Zucker entstanden, oder in Procenten 110,76.

In der nachfolgenden Tabelle sind die eben besprochenen Versuche der besseren Uebersicht wegen zusammengestellt.

\begin{tabular}{c|c|c|c|c|c}
\hline \hline $\begin{array}{c}\text { Num. } \\
\text { des } \\
\text { Vers. }\end{array}$ & $\begin{array}{c}\text { Angew. } \\
\text { lufttrockene } \\
\text { Stärke. }\end{array}$ & $\begin{array}{c}\text { Absolut } \\
\text { reine } \\
\text { Stärke. }\end{array}$ & $\begin{array}{c}\text { spec. Gew. } \\
\text { minus } \\
\text { Salzsäure. }\end{array}$ & $\begin{array}{c}\text { Traubenz. } \\
\text { in } 100 \text { Ccm. } \\
\text { Lös.in Grm. }\end{array}$ & $\begin{array}{c}\text { Procente } \\
\text { Traubenz. }\end{array}$ \\
\hline \hline I. & 31,767 & 25 & 1,0426 & 11,18 & 111,8 \\
II. & 31,767 & 25 & 1,04258 & 11,176 & 111,76 \\
III. & 31,767 & 25 & 1,0424 & 11,128 & 111,28 \\
IV. & 31,767 & 25 & 1,0422 & 11,076 & 110,76
\end{tabular}

Aus diesen Versuchen ergiebt sich ein mittleres spec. Gewicht von 1,0424 und in $100 \mathrm{Ccm}$. Lösung ein Zuckergehalt von 11,14 Grm. (oder auf 100 Theile Stärke 111,4 
320 Schulze: Die elementare Zusammensetzung

Theile Traubenzucker) während die Theorie einen Zuckergehalt von $11,11 \mathrm{Grm}$. und mithin ein spec. Gewicht von 1,0423 verlangt.

Diese Versuche beweisen ebenfalls, dass die Verzuckerung der Stärke gemäss der Gleichung:

verläuft.

$$
\mathrm{C}_{6} \mathrm{H}_{10} \mathrm{O}_{5}+\mathrm{H}_{2} \mathrm{O}=\mathrm{C}_{6} \mathrm{H}_{12} \mathrm{O}_{6}
$$

C. Durch Verzuckerung mittelst Salzsäure und Bestimmung des Traubenzuckers durch Polarisation.

Durch in neuerer Zeit angestellte Beobachtungen über das Verhalten des Traubenzuckers gegen polarisirtes Licht haben Tollens, Brown und Heron, Salomon u. A. nachgewiesen, dass dem Traubenzucker eine spec. Drehung von $58,68^{\circ}$, auf die Uebergangsfarbe $(\alpha j$.) bezogen, zukommt, d. h. $10 \mathrm{Grm}$. Traubenzucker zu $100 \mathrm{Ccm}$., bei 17,50 gelöst, vermögen dem Strahle $u$ j. eine Ablenkung von $58,68^{\circ} \mathrm{zu}$ geben.

Meine sämmtlichen Bestimmungen wurden mit einem von Schmidt und Haentsch in Berlin stammenden Soleil-Ventzke-Scheiblerschen Apparate ausgefübrt, der mit einem Wild'schen Polaristrobometer verglichen war 100 Theile der Scala des von mir angewandten Apparates entsprechen 38,4 Bogengraden $\alpha j$. Der Werth für $\alpha j$ ist dann bei allen Bestimmungen nach der Formel:

$$
\alpha j=\frac{a \times 0,384 \times 100}{1 \times \mathrm{p}}
$$

ermittelt worden, worin $a \mathrm{j}$ die spec. Drehung, $a$ die am Soleil-Ventzke-Scheibler beobachteten Grade, 1 die Länge des Beobachtungsrohres in Decimetern, p. das Gewicht der in $100 \mathrm{Ccm}$. der Lösung enthaltenen Trockensubstanz bedeutet.

Durch zahlreiche Versuche haben Herr Dr. Salomon und ich gefunden, dass $10 \mathrm{Grm}$. Traubenzucker zu $100 \mathrm{Ccm}$. gelöst (in Wasser) in unserem Apparate eine Drehung von $30,6^{0}$ verursachten. Diese Zahl haben wir nun zur Aufstellung von zwei Tabellen benutzt, mittelst deren man mit Leichtigkeit aus den gefundenen Graden den entsprechenden 
Traubenzucker und umgekehrt, aus einer bekannten. Menge Traubenzucker die entsprechenden Polarisationsgrade berechnen kann.

Tabelle zur Ermittelung der Grammewasserfreien Traubenzuckers in $100 \mathrm{Ccm}$. Lösung aus den gefundenen Graden Soleil-Ventzke-Scheibler.

\begin{tabular}{|c|c|c|c|c|c|}
\hline $\begin{array}{c}\text { Abgeles. } \\
\text { Grade. }\end{array}$ & $\begin{array}{c}\text { In } 100 \text { Cem. } \\
\text { Lös. enth. } \\
\text { Traubenanck. } \\
\text { in Grm. }\end{array}$ & $\begin{array}{c}\text { Abgeles. } \\
\text { Grade. }\end{array}$ & $\begin{array}{l}\text { In } 100 \mathrm{Ccm} . \\
\text { Lös. enth. } \\
\text { Traubenzuek. } \\
\text { in Grm. }\end{array}$ & $\begin{array}{l}\text { Abgeles. } \\
\text { Grade. }\end{array}$ & $\begin{array}{l}\text { In } 100 \mathrm{Ccm} . \\
\text { Lös. enth. } \\
\text { Traubenzuck. } \\
\text { in Grm. }\end{array}$ \\
\hline 1 & 0,3268 & 5 & 1,634 & 9 & 2,9412 \\
\hline 2 & 0,6536 & 6 & 1,9608 & 10 & 3,268 \\
\hline 3 & 0,9804 & 7 & 2,2876 & & \\
\hline 4 & 1,3072 & 8 & 2,6144 & & \\
\hline
\end{tabular}

Tabelle zur Ermittelung der Bogenrade aus der bekannten Menge wasserfreien Traubenzuckers.

\begin{tabular}{c|c|c|c|c|c}
\hline $\begin{array}{c}\text { Trbnz. } \\
\text { in Grm. }\end{array}$ & $\begin{array}{c}\text { Grade am } \\
\text { Soleil-Ventzke-Ver } \\
\text { Scheibler. }\end{array}$ & $\begin{array}{c}\text { Trbnz. } \\
\text { in Grm. }\end{array}$ & $\begin{array}{c}\text { Grade am } \\
\text { Soleil-Ventzke- } \\
\text { Scheibler. }\end{array}$ & $\begin{array}{c}\text { Trbnz. } \\
\text { in Grm. }\end{array}$ & $\begin{array}{c}\text { Grade am } \\
\text { Soleil-Ventzke- } \\
\text { Scheibler. }\end{array}$ \\
\hline $\mathbf{1}$ & 3,06 & 5 & 15,3 & 9 & 27,54 \\
2 & 6,12 & 6 & 18,36 & 10 & 30,6 \\
3 & 9,18 & 7 & 21,42 & & \\
4 & 12,24 & 8 & 24,48 & &
\end{tabular}

In Folgendem sind die Resultate, welche ich durch die Polarisation erhalten habe, wiedergegeben.

Versuch I. $31,43 \mathrm{Grm}$. lufttrockener Stärke (entsprechend 24,736 absolut reiner Stärke) wurden mit $20 \mathrm{Ccm}$. Salzsäure vom spec. Gew. 1,125 und $180 \mathrm{Ccm}$. Wasser 3 Stunden am Rückflusskühler gekocht, darauf zu $250 \mathrm{Ccm}$. aufgefüllt und danu polarisirt.

Beobachtet im $200 \mathrm{Mm}$. Rohr $33,8^{\circ}$ S.V.S. Nach obiger Tabelle berechneter Zuckergehalt in $100 \mathrm{Ccm} .=11,0458 \mathrm{Grm}$. In Procenten ausgedrückt $=111,6$. Zur Bestimmung der spec. Drehung wurde mittelst des spec. Gewichts der Trockengehalt der Lösung festgestellt und darauf nach der oben angegebenen Formel der Werth für $\alpha \mathrm{j}$ berechnet.

Gef. spee. Gewicht 1,0422=11,076 Grm. Trockensubst. in $100 \mathrm{Ccm}$. $\alpha \mathbf{j}=58,591$.

Versuch II. Ist in derselben Weise angestellt, wie Versuch I.

Beobachtete Polarisation im $200 \mathrm{Mm}$. Rohr $=39,8^{\circ}$ S.V.S. Berechneter Zuckergchalt $=11.0458$, in Procenten ausgedrückt 111,6\%. Gef. spec. Gewicht $1,0421=11,049 \mathrm{Grm}$. Trockensubst. in $100 \mathrm{Ccm} . \alpha \mathrm{j}=$ 58,734 . 


\section{Schulze: Die elementare Zusammensetzung}

Versuch III. In derselben Weise angestelt wie Versuch I.

Beobachtete Polarisation im $200 \mathrm{Mm}$. Rohr $=34^{0}$ S.V.S., daraus berechneter Trockengehalt in $100 \mathrm{Ccm} .=11,112 \mathrm{Grm}$, in Procenten ausgedrückt $=112,3 \%$. Gef, spec. Gewicht 1,0423 $=11,11$ Grm. Trockensubstanz in $100 \mathrm{Ccm} . \alpha \mathrm{j}=58,757$.

Versuch IV. In derselben Weise angestellt, wie Versuch I.

Beobachtete Polarisation im $200 \mathrm{Mm}$. Rohr $=33,9^{\circ}$ S.V.S., daraus berechneter Zuckergehalt in $100 \mathrm{Ccm} .=11,0756$ Grm., in Procenten ausgedrückt $=111,9 \%$. Gef. spec. Gewicht $1,0422=11,076$ Grm. Trockensubstanz in 100 Ccm. $\alpha j=58,59$.

In der nachfolgenden Tabelle habe ich die eben beschriebenen Versuche zusammengestellt.

\begin{tabular}{|c|c|c|c|c|c|c|}
\hline $\begin{array}{c}\text { Nurn. } \\
\text { des } \\
\text { Vers. }\end{array}$ & $\begin{array}{l}\text { Angew. } \\
\text { lufttr. } \\
\text { Stärke. } \\
\text { in Grm. }\end{array}$ & $\begin{array}{l}\text { Absolut } \\
\text { reine } \\
\text { Stärke } \\
\text { in Grm. }\end{array}$ & $\begin{array}{l}\text { Beob. } \\
\text { Polaris. } \\
\text { im } 200 \\
\text { Mm.-R. }\end{array}$ & $\begin{array}{l}\text { Berechnet. } \\
\text { Traubenz. } \\
\text { in } 100 \mathrm{Ccm} .\end{array}$ & $\begin{array}{l}\text { Procente } \\
\text { Traubenz. }\end{array}$ & $\alpha \mathbf{j}$. \\
\hline I & 31,43 & 24,736 & $33,8^{0}$ & 11,0458 & 111,6 & 58,591 \\
\hline II & 31,43 & 24,736 & $33,8^{0}$ & 11,0458 & 111,6 & 58,734 \\
\hline III & 31,43 & 24,736 & $34^{0}$ & 11,112 & 112,3 & 58,757 \\
\hline IV & 31,43 & 24,736 & $33,9^{\circ}$ & 11,0756 & 111,9 & 58,591 \\
\hline
\end{tabular}

Hieraus ergiebt sich ein mittlerer Procentgehalt von $111,85 \%$ Traubenzucker und in $100 \mathrm{Ccm}$. Lösung ein Zuckergehalt von 11,0698 Grm. $\alpha$ j ergiebt sich im Mittel zu 58,668 statt 58,63. Auch diese Versuche zeigen zur Genüge, dass aus 100 Theilen Stärke bei der Verzuckerung 111,11 Theile Traubenzucker gebildet werden. Die Umwandlung der Stärke in Traubenzucker also nach der Formel vor sich geht.

$$
\mathrm{O}_{6} \mathrm{H}_{10} \mathrm{O}_{5}+\mathrm{H}_{2} \mathrm{O}=\mathrm{C}_{6} \mathrm{H}_{12} \mathrm{O}_{6}
$$

D. Elementaranalyse der Weizenstärke.

Obgleich schon eine grosse Reihe Elementaranalysen über die Stärke von den verschiedensten Seiten vorliegen, so weichen diese dennoch ganz erheblich von einander $\mathbf{a b}$, und darf ich es daher nicht unterlassen, auch an dieser Stelle darauf zurückzukommen.

Der grösste Theil der ausgeführten Elementaranalysen bezieht sich auf bei $100^{\circ}$ getrocknete Stärke.

Da nun diese Temperatur zum Trocknen derselben, wie schon oben angeführt, nicht ausreicht, so scheint mir hierin 
der Grund zu liegen, weshalb die Analysen so von einander abweichen.

So fand Prout z. B. in einer Elementaranalyse der Weizenstärke: lufttrocken: $\quad 37,5 \% \mathrm{C} 6,94 \% \mathrm{H} \mathrm{55,56 \% \textrm {O }}$

bei $100^{\circ}$ getrock. $42,8,6,35, \quad 50,85 "$ $" 140^{\circ} \quad, \quad 44,0 \quad, \quad 6,2 \quad " 49,8 \quad "$

Marcet bei $100^{\circ}$ getrock. $43,7 \%$ C $6,7 \% \mathrm{H} \mathrm{49,6 \%} \mathrm{O}$

Dean $\left." 100^{\circ} \quad, \quad 44,0,, 6,53,49,43,{ }^{1}\right)$

Sämmtliche Verbrennungen wurden mit gut getrocknetem Sauerstoff ausgeführt, welcher nachher durch trockene und von Kohlensäure befreite Luft verdrängt wurde.

Probe I. 0,4775 Grm. lufttrockener Stärke $=0,38$ Grm. absolut reiner.

Gef. $\mathrm{CO}_{2}=0,621$ Grm., $\mathrm{H}_{2} \mathrm{O}=0,3065$ nach Abzug anhaft. $=0,21$ Grm. Berechnung: $44,21 \% \mathrm{C}, 5,868 \% \mathrm{H}, 49,922 \% 0$.

Probe II. 0,488 Grm. luftrock. Stärke $=0,384$ Grm. absolut reiner.

Gef. $\mathrm{CO}_{2}=0,6315$ Grm., $\mathrm{H}_{2} \mathrm{O}=0,3075$ Grm. nach Abzug anhaft. $=$ 0,2085 Grm. Berechnang: 44,79\% C, 6,041\% H, 49,169\% $\mathrm{O}$.

Probe IIJ. $0,5195 \mathrm{Grm}$. lufttr. $=0,4088 \mathrm{Grm}$. absolut reiner Stärke.

Gef. $\mathrm{CO}_{2}=0,6685$ Grm., $\mathrm{H}_{2} \mathrm{O}=0,327$ Grm. nach $\mathrm{Alozug}$ anhaft. $0,223 \mathrm{Grm}$. Berechnung: 44,681\% C, 5,87\% H, 49,449\% O.

Probe IV. $0,554 \mathrm{Grm}$. luftrock $=0,436 \mathrm{Grm}$. absolut reiner Stärke.

Gef. $\mathrm{CO}_{2}=0,717$ Grm., $\mathrm{H}_{2} \mathrm{O}=0,353$ Grm. nach Abzug anhaft. $=$ 0,2616 Grm. Berechnung: 44,839\% $\mathrm{C}, 6,651 \% \mathrm{H}, 48,41 \% \mathrm{O}$.

Probe V. 0,633 Grm. Infttrock $=0,498 \mathrm{Grm}$. absolut reiner Stärke.

Gef. $\mathrm{CO}_{2}=0,8175$ Grm., $\mathrm{H}_{2} \mathrm{O}=0,406$ nach Abzug anhaft. $=0,2786 \mathrm{Grm}$. Berechnung: $44,718 \% \mathrm{C}, 6,205 \% \mathrm{H}, 49,077 \% \mathrm{O}$.

Probe VI. 0,615 Grm. lufttrock. $=0,484 \mathrm{Grm}$. absolut reiner Stärke.

Gef. $\mathrm{CO}_{2}=0,786$ Grm., $\mathrm{H}_{2} \mathrm{O}=0,374$ Grm. nach Abzug anbaft. $=$ 0,251 Grm. Berechnong: 44,277\% C, 5,785\% $\mathrm{H}, 49,938 \% \mathrm{O}$.

Aus diesen sechs Verbrennungen ergiebt sich ein Mittel von $44,573 \% \quad \mathrm{C}, 6,07 \% \mathrm{H}$ und $49,344 \% \mathrm{O}$.

Die Verbindung $\mathrm{C}_{6} \mathrm{H}_{10} \mathrm{O}_{5}$ enthält:

Berechnet. Gefunden. Die Verb. $\mathrm{C}_{36} \mathrm{H}_{62} \mathrm{O}_{31}$ enthält:

$\begin{array}{rrrrr}44,44 \% \mathrm{C} & 44,573 \% & \mathrm{C} & 43,636 \% & \mathrm{C} \\ 6,172, \mathrm{H} & 6,07, & \mathrm{H} & 6,262, & \mathrm{H} \\ 49,382, \mathrm{O} & 49,344, & \mathrm{O} & 50,102, & \mathrm{O}\end{array}$

Man ersieht hieraus, dass die gefundenen Zahlenwerthe

1) Diese Zahlen sind Gmelin-Kraut, org. Chemie 4. Aufl. Bd. 4, Abthlg. I, S. 538 entnommen. 


\section{Schulze: Die elementare Zusammensetzung}

mit denen nach der Formel $\mathrm{C}_{6} \mathrm{H}_{10} \mathrm{O}_{5}$ berechneten fast genau übereinstimmen.

Aus den bei diesen Untersuchungen erhaltenen Resultaten glaube ich nun mit voller Sicherheit die der Weizenstärke zukommende chemische Zusammensetzung ableiten zu dürfen und somit die Frage: "Ist die Weizenstärke nach der Formel $\mathrm{C}_{6} \mathrm{H}_{10} \mathrm{O}_{5}$ oder ist dieselbe nach der Formel $\mathrm{C}_{36} \mathrm{H}_{62} \mathrm{O}_{31}$ zusammengesetzt?" zum endgültigen Abschluss zu bringen.

Nehmen wir an, dass die von Naegeli und Sachsse für die Stärke angenommene Formel $\mathrm{C}_{34} \mathrm{H}_{62} \mathrm{O}_{31}$ diejenige sei, welche sich den Thatsachen am Besten anschliesse, so müssten bei der Ueberführung der Stärke in Traubenzucker aus 100 Theilen reiner Stärke 109,09 Theile Traubenzucker oder aus 990 Theilen reiner Stärke 1080 Theile Traubenzucker entstehen, mit anderen Worten, die Umwandlung der Stärke in Traubenzucker müsste nach der Gleichung:

verlaufen.

$$
\mathrm{C}_{36} \mathrm{H}_{63} \mathrm{O}_{31}+5 \mathrm{H}_{2} \mathrm{O}=6 \mathrm{C}_{6} \mathrm{H}_{13} \mathrm{O}_{6}
$$

Mit diesen Zahlen stehen nun aber, wie wir gesehen haben, die von mir bei der Verzuckerung der Weizenstärke gefundenen durchaus nicht im Einklange, sondern zeigen vielmehr, dass bei der Verzuckerung der Weizenstärke aus 100 Theilen reiner Stärke 111,11 Theile Traubenzucker oder aus 162 Theilen reiner Stärke 180 Theile Traubenzucker entstehen, und mithin die Umwandlung der Weizenstärke in Traubenzucker nach der Gleichung:

vor sich geht.

$$
\mathrm{C}_{6} \mathrm{H}_{10} \mathrm{O}_{5}+\mathrm{H}_{2} \mathrm{O}=\mathrm{C}_{6} \mathrm{H}_{12} \mathrm{O}_{6}
$$

Da nun ausserdem, wie schon oben gezeigt, auch die von mir bei der Elementaranalyse erhaltenen Zahlen nicht mit den nach der Formel $\mathrm{C}_{36} \mathrm{H}_{62} \mathrm{O}_{31}$ berechneten übereinstimmen, wohl aber mit denen, welche nach der Formel $\mathrm{C}_{6} \mathrm{H}_{10} \mathrm{O}_{5}$ berechnet sind, so scheint es mir demnach keinem Zweifel mehr zu unterliegen, dass die Weizenstärke nach der Formel $\mathrm{C}_{6} \mathrm{H}_{10} \mathrm{O}_{5}$ zusammengesetzt ist.

\section{Einwirkung verdünnter Essigsäure auf Stärke.}

Beim weiteren Verfolg meiner Untersuchungen über die Stärke und deren Umwandlungsproducte durch chemische 
Agentien wurde ich dazu geführt, die durch Behandeln der Stärke mit verdünnten organischen Säuren entstehenden Körper zu untersuchen. Bei Durchsicht der Literatur über diesen Punkt fiel es mir auf, dass über die Einwirkung der Essigsäure von mittlerer Concentration auf Stärke nur wenige und zum Theil ganz widersprechende Angaben vorliegen. So erhielt z. B. Béchamp ${ }^{1}$ ) durch 1- bis 2 stündiges Erhitzen von Stärkemehl mit Essigsäure (bei $100^{\circ}$ ) in zugeschmolzenen Röhren eine Flüssigkeit von grossem Rotationsvermögen, die durch Jod blau gefärbt wurde und mit Alkohol einen Niederschlag gab; nach längerem Erhitzen (12 bis 28 Stunden) auf $100^{\circ}$ bis $130^{\circ}$ hatte die Flüssigkeit an Rotationsvermögen verloren, gab mit Alkohol keine Füllung mehr und hinterliess beim Eindampfen einen nicht gährungsfähigen Rückstand. Mit dieser Angabe stimmen die von Kirchhoff und Biot ${ }^{2}$ ) gemachten überein, während nach den Angaben von Persoz ${ }^{3}$ ) durch Behandeln der Stärke mit wässriger Essigsäure erst Dextrin und dann Zucker entsteht.

Da nun diese Daten auch noch zum grössten Theil einer früheren Zeit angehören, so erschien es mir geboten, die Frage: „Welche Umwandlungsproducte entstehen durch Behandeln der Stärke mit mässig concentrirter Essigsäure?" einer näheren Betrachtung zu unterwerfen.

Durch eine Reihe von Versuchen, welche mit Essigsäure von verschiedener Stärke ausgeführt wurden, suchte ich zunächst diejenige Concentration zu ermitteln, bei welcher voraussichtlich die besten Resultate zu erwarten waren. Dieselben führten mich auch alsbald zu der Ueberzeugung, dass es für meine Zwecke am Entsprechendsten sei, eine 20 proc. Säure zu verwenden, und ist diese Concentration auch bei allen Versuchen innegehalten.

Zur Ausfuhhrung sämmtlicher Versuche bediente ich mich einer Reisstärke, von welcher 120,71 Grm. lufttrockener Stärke genau 100 Grm. absolut reiner Stärlke entsprechend

1) Dies. Journ. 69, 548.

9) Compt. rend. 17, 1064.

3) Gmelin-Kraut, org. Chemie 4. Aufl. Bd. 4 Abthlg. I, S. 545. 


\section{Schulze: Die elementare Zusammensetzung}

waren. Bei jedem Versuch wurde stets diejenige Menge Stärke angewandt, welche erforderlich war, um eine annähernd 10proc. Lösung zu erhalten.

Versuch 1. Zu demselben wurden $25 \mathrm{Grm}$. absolut reiner Stärke, entsprechend 30,17 Grm. lufttrockener, angewandt. Diese 30,17 Grm. wurden 10 Stunden lang im Kochsalzbade mit einer 20\% Essigsäure (166,60 Grm. 30\% Essigsäure zu $250 \mathrm{Ccm}$.) am Rückflusskühler erhitzt, alsdann zu $250 \mathrm{Ccm}$. verdünnt und darauf in der schon erwähnten Art und Weise das spec. Gewicht der Lösung ermittelt. Dieses ergab sich nach Abzug des spec. Gewichts einer $20 \%$ Essigsäure zu 1,0364. Daraus berechnet sich unter: Zugrundelegung des Factors 3,62 (das von mir für das Dextrin $c$ ermittelte spec. Gewicht siehe Versuch III) ein Trockengehalt von 10,055 Grm. in $100 \mathrm{Ccm}$. Lösung.

Die Polarisation dieser Lösung betrug im $200 \mathrm{Mm}$. Rohr 103,5 S.V.S. Die spec. Drehung der Substanz beträgt demnach $\alpha \mathrm{j}=197,633^{\circ}$. (Die spec. Drehung ist nach der Formel $\alpha \mathrm{j}=\frac{\alpha \times 0,384 \times 100}{1 \times \mathrm{p}}$ berechnet.)

Die Lösung gab mit Jod eine roth-violette Färbung, mit Alkohol einen weissen Niederschlag und reducirte Fehling'sche Lösung.

Versuch II. Hierbei wurden $25 \mathrm{Grm}$. absolut reiner, gleich 30,17 Grm. lufttrockener Stärke 8 Stunden lang mit einer 20proc. Essigsäure in der Bombe im Kochsalzbade erhitzt, alsdann zu $250 \mathrm{Ccm}$. aufgefüllt und dann das spec. Gewicht, und die Polarisation der Lösung bestimmt.

Gefundenes spec. Gewicht nach Abzug desjenigen der Essigsäure $=1,0372$. Daraus berechneter Trockengehalt = $10,276 \mathrm{Grm}$. in $100 \mathrm{Ccm}$. Lösung. Beobachtete Drehung= $104^{0}$ S.V.S.; hieraus berechnet sich eine spec. Drehung von $\alpha \mathrm{j}=194,317^{\circ}$.

Die Lösung wurde durch Jod ebenfalls roth-violett gefärbt, gab mit Alkohol einen Niederschlag und reducirte Fehling'sche Lösung. -

Diese beiden Versuche hatten nur den Zweck zu ermitteln, 
wie weit die Umwandlung der Stärke durch Essigsäure vor sich gegangen und was im Wesentlichen für Producte entstanden waren. In dem folgenden Versuche suchte ich nun zunächst den durch Alkohol fällbaren Körper zu erhalten und darauf die Eigenschaften desselben festzustellen.

\section{Darstellung des Dextrins.}

Versuch III. Zu diesem Versuche wurden drei Bomben, von denen jede mit 25 Grm. reiner Stärke $(30,17 \mathrm{Grm}$. lufttrockener) und einer 20procent. Essigsäure beschickt war, 7 Stunden unter Druck im Kochsalzbade erhitzt, darauf der Inhalt einer jeden Bombe zu $250 \mathrm{Ccm}$. verdünnt und abfiltrirt.

Die klare Lösung wurde nun mit absolutem Alkohol versetzt, und so ein schleimiger Körper ausgeschieden, der sich schnell auf dem Boden des Gefässes absetzte. Um denselben ganz von Essigsäure und sonstigen Verunreinigungen zu befreien, wurde derselbe wiederholt in Wasser gelöst und mit Alkohol ausgefällt. Nachdem diese Operation viermal wiederholt war, wurde der Körper über Schwefelsäure getrocknet und konnte schon nach einigen Tagen zu einem feinen weissen Pulver zerrieben werden. (Aus $25 \mathrm{Grm}$. Stärke wurden ungefähr 15-20 Grm. reines Dextrin erhalten.)

Die genauere Untersuchung dieses Körpers ergab, dass derselbe vollständig identisch ist mit der von Bondonneau ${ }^{1}$ ) als Dextrin $\approx$ bezeichneten Substanz. Nach den Angaben dieses Chemikers wird das Dextrin $\propto$ durch Jod roth gefärbt, durch Alkohol aus seinen Lösungen ausgefällt und besitzt endlich eine spec. Drehung von $\alpha(D)=186^{\circ}$. Rechnet man die spec. Drehung von $\alpha \mathrm{D}$ auf $\alpha \mathrm{j}$ nach dem von Brown und Heron ${ }^{2}$ ) angegebenen Verhältnisse

$$
\begin{aligned}
\alpha \mathrm{j}: \alpha \mathrm{D} & =24: 21,54 \\
\text { oder } \alpha \mathrm{D}: \alpha \mathrm{j} & =1: 1,1124
\end{aligned}
$$

um, so erhält man eine spec. Drehung von $\alpha j=207,24^{\circ}$ eine Zahl, welche, wie wir gleich sehen werden, fast genau mit der von mir für den Strahl $\alpha$ j ermittelten übereinstimmt.

1) Ber. Berl. chem. Gesell. 9, 61 und 69.

2) Ann. d. Chem. u. Pharm. 199 S. 179 Anm. 


\section{Schulze: Die elementare Zusammensetzung}

Probe I. 4,821 Grm. Substanz sind zu $50 \mathrm{Ccm}$. gelöst. Das spec. Gewicht dieser Lösung betrug 1,03449; rechnen wir dieses auf $10 \mathrm{Grm}$. der Substanz, zu 100 Cem. gelöst, um, so erhalten wir ein spec. Gew. von 1,0358. Die beobachtete Drehung betrug $51,8^{\circ}$ S.V.S. im $100 \mathrm{Mm}$. Rohr; es berechnet sich daraus nach der Formel $\alpha \mathrm{j}=\frac{\alpha \times 0,384 \times 100}{1 \times \mathrm{p} .}$ eine spec. Drehung von $206,297^{\circ} \alpha \mathrm{j}$.

Probe II. 4,319 Grm. Substanz sind za 50 Ccm. gelöst. Das gefundene spec. Gewicht betrug 1,03161; auf $10 \mathrm{Grm}$. in $100 \mathrm{Ccm}$. berechnet: 1,0366 .

Im $100 \mathrm{Mm}$.-Rohr wurde eine Drehung von $46,8^{\circ}$ S.V.S. beobachtet, die daraus berechnete Drehung beträgt $\alpha \mathrm{j}=208,048^{\circ}$.

Probe III. 4,5465 Grm. Substanz sind zu 50 Ccm. gelöst. Das gefundene spec. Gewicht betrug 1,03291 ; auf $10 \mathrm{Grm}$. in $100 \mathrm{Ccm}$. berechnetes spec. Gewicht $=1,0362$.

Beobachtete Drehung in $100 \mathrm{Mm}$.-Rohr $49^{\circ}$ S.V.S. Daraus berechnete Drehung für $\alpha j=207,104^{\circ}$.

Aus diesen drei Bestimmungen ergiebt sich für 10 Grm. Dextrin, zu $100 \mathrm{Ccm}$. gelöst, im Mittel ein spec. Gewicht von 1,0362 und eine spec. Drehung ron $\alpha j=207,149^{\circ}$, welcher Werth von dem, nach den Angaben Bondonneau's, für Dextrin e berechneten $\alpha j=207,24^{\circ}$ nur um 0,091 abweicht.

Die bei der Elementaranalyse dieses Körpers erhaltenen Zahlen stimmen ebenfalls mit denen, welche die für das Dextrin angenommene Formel $\mathrm{C}_{6} \mathrm{H}_{10} \mathrm{O}_{5}$ verlangt.

Probe I. Angewandt sind 0,222 Grm. Substanz.

Gef. $\mathrm{CO}_{2}=0,3615 \mathrm{Grm}$, berechneter $\mathrm{C}=44,369$

$$
\begin{array}{rll}
\mathrm{H}_{2} \mathrm{O}=0,126 \quad \text { " } & \mathrm{H}=6,3063 \\
\mathrm{O} & =49,3247
\end{array}
$$

Probe II. Angewandt sind 0,2885 Grm. Substanz.

Gef. $\mathrm{CO}_{2}=0,4705$ Grm, berechneter $\mathrm{C}=44,402$

$$
\text { " } \begin{aligned}
\mathrm{H}_{2} \mathrm{O}=0,1685 \quad " \quad, \quad \mathrm{H} & =6,828 \\
\mathrm{O} & =48,77
\end{aligned}
$$

Probe III. Angewandt sind 0,1847 Grm. Substanz.

Gef. $\mathrm{CO}_{2}=0,301$ Grm., berechneter $\mathrm{C}=44,402$

$$
\begin{array}{rlrl} 
& \mathrm{H}_{2} \mathrm{O}=0,1065 \quad \text { " } & & \mathrm{H}=6,341 \\
\mathrm{O} & =49,257
\end{array}
$$

Aus diesen drei Verbrennungen ergiebt sich im Mittel eine proc. Zusammensetzung von $44,391 \% \mathrm{C}$, die Formel verlangt $44,44 \% \mathrm{C}$

$$
\begin{aligned}
& 6,491 \% \mathrm{H} \quad, \quad, \quad, \quad 6,172 \% \mathrm{H} \\
& 49,118 \% \text { O } ", \quad 49,382 \% 0
\end{aligned}
$$

Dass der Wasserstoffgehalt in Aualyse II etwas zu 
hoch ausgefallen ist, findet seine Erklärung dadurch, dass die Substanz vorher nicht genügend getrocknet war.

Die Auflösung des von mir erhaltenen Körpers wurde durch Jod roth gefärbt. Fehling'sche Lösung wurde durch denselben nicht reducirt.

Da die Eigenschaften des Körpers, welcher mir zur Verfügung stand, vollkommen mit denen übereinstimmen, welche Bondonneau an seinem Dextrin $\alpha$ beobachtet hatte, so unterliegt es wohl keinem $Z_{\text {weifel }}$ mehr, dass dieselben vollkommen identisch sind. Da nun sämmtliche Lösungen, welche ich durch Einwirkung von Essigsäure auf Stärke erhielt, Fehling'sche Lösung, je nachdem die Einwirkung der Säure von längerer oder kürzerer Zeitdauer gewesen war, mehr oder weniger reducirten, diese Reduction aber von dem gebildeten Dextrin nicht herrührte, so handelte es sich jetzt darum, den Körper zu bestimmen, durch welchen die Reduction herbeigeführt wurde. Ausserdem hatte ich anch an den erhaltenen Lösungen die Beobachtung gemacht, dass mit zunehmendem Reductionsvermögen die Lösungen an Rotationsvermögen verloren.

Es waren dies Erscheinungen, welche mit Bestimmtheit annehmen liessen, dass die Einwirkung der Essigsäure auf Stärkemehl nicht nur auf einer blossen Umwandlung desselben in Dextrin beruhe, sondern eine weitergehende Umwandlung der Stärke zur Folge habe.

In der That habe ich durch Versuche gefunden, dass bei fortgesetzter Einwirkung von Essigsäure auf Sïärkemehl Traubenzucker gebildet wird, und es ist mir, wenn auch mit grossen Schwierigkeiten, gelungen, denselben isolirt darzustellen. Die zunächst folgenden Versuche sind bestimmt, zu beweisen, dass durch Einwirkung von Essigsäure aut Stärke überhaupt Traubenzucker gebildet wird.

Versuch IV. Es wurden 25 Grm. reiner Stärke (30,17 Grm. luftrockener) angewandt. Diese wurden mit einer 20 proc. Essigsäure 4 Stunden im Kochsalzbade erhitzt und alsdann zu $250 \mathrm{Ccm}$. aufgefüllt.

Das spec. Gewicht dieser Lösung wurde nach Abzug des einer 20 procent. Essigsäure entsprechenden zu 1,0304 


\section{Schulze: Die elementare Zusammensetzung}

gefunden. Daraus berechnet sich ein Trockengehalt von 8,398 Grm. pro $100 \mathrm{Ccm}$. Lösung.

Die beobachtete Drehung betrug $87,2^{\circ}$ S.V.S. Daraus berechnet sich für $\alpha$ j eine Drehung von 199,361 .

Durch Jod wurde die Lösung violett gefärbt. Zur Bestimmung des gebildeten Traubenzuckers wurden $55 \mathrm{Ccm}$. der Lösung = 4,618 Grm. Trockensubstanz zu $250 \mathrm{Ccm}$. gelöst, und nach Allihn's Methode mit alkalischer Kupferlösung behandelt. Reducirt waren 0,365 Grm. Kupfer. 1 Grm. Trockensubstanz entspricht demnach 0,079 Grm. Kupfer. Da nun nach Allihn 1,852 Grm. Kupfer 1 Grm. Dextrose entsprechend sind, so entsprechen diese 0,079 Grm. Kupfer 0,0426 Grm. Traubenzucker. In $10 \mathrm{Grm}$. des Gemisches sind demnach 0,426 Grm. Traubenzucker und 10$0,426=9,574$ Grm. Dextrin enthalten.

Da nun 10 Grm. Traubenzucker zu $100 \mathrm{Ccm}$. gelöst eine Drehung von $\alpha j=58,68^{\circ}$ bewirken, so beträgt die Drehung von 0,426 Grm. Traubenzucker 2,505 $\mathrm{cj}$.

$10 \mathrm{Grm}$. Dextrin $\alpha$, zu $100 \mathrm{Ccm}$. gelöst, besitzen, wie ich eben gezeigt habe, eine spec. Drehung von $207,149^{\circ}(\alpha \mathrm{j})$.

Es müssten demnach 9,573 Grm. Dextrin eine Drehung von $198,312^{\circ}(\alpha \mathrm{j})$ hervorrufen. Die gesammte berechnete Drehung von 10 Grm. des Gemisches beträgt demnach $198,312^{0}+2,505^{0}=200,83^{0} \quad(a j)$, statt der gefundenen $199,361^{\circ}(\alpha \mathrm{oj})$.

Versuch V. Es wurden ebenfalls 25 Grm. absolut reiner Stärke (entsprechend 30,17 Grm. lufttrockener) mit 20 proc. Essigsäure in der Bombe im Kochsalzbade erhitzt und zwar 6 Stunden lang. Darauf wurde die Flüssigkeit zu $250 \mathrm{Ccm}$. aufgefüllt.

Das spec. Gewicht der Lösung betrug 1,0315. Darans berechnet sich ein Trockengehalt von 8,702 Grm. für $100 \mathrm{Ccm}$. Lösung. Im $200 \mathrm{Mm}$.-Rohre wurde eine Drehung von 89,3 $3^{\circ}$ beobachtet. Daraus berechnet sich für $\alpha$ j eine spec. Drehung von $197,03^{\circ}$. $55 \mathrm{Ccm}$. der Lösung, gleich 4,786 Grm. Trockensubstanz, wurden zu $250 \mathrm{Ccm}$. verdümmt und reducirten diese $0,49 \mathrm{Grm}$. Kupfer. $1 \mathrm{Grm}$. Trockensubstanz entspricht demnach 0,102 Grm. Kupfer. Da 1,852 Grm. Kupfer = 
1 Grm. Traubenzucker sind, so entsprechen diese 0,102 Grm. Kupfer 0,05508 Grm. Traubenzucker.

In $10 \mathrm{Grm}$. des Gemisches sind demnach 0,5508 Grm. Traubenzucker und 9,45 Grm. Dextrin enthalten.

$10 \mathrm{Grm}$. Traubenzucker verlangen eine Drehung von $58,68^{\circ}$. $0,5508 \mathrm{Grm}$. demnach eine solche von $3,227^{\circ}(\alpha \mathrm{j})$. $10 \mathrm{Grm}$. Dextrin $*$ drehen $207,149^{\circ}$. 9,45 Grm. demnach 195,755 .

Die berechnete Gesammtdrehung beträgt $198,982^{\circ}(\alpha j)$, statt der gefundenen $197,03^{\circ}(\alpha \mathrm{j})$.

Versuch VI. 25 Grm. reiner Stärke (30,17 Grm. lufttrock.) sind mit einer 20 proc. Essigsäure 8 Stunden im Kochsalzbade in der Bombe erhitzt, darauf zu $250 \mathrm{Ccm}$. aufgefüllt.

Das spec. Gewicht der Lösung betrug 1,0337 und berechnet sich daraus ein Trockengehalt von 9,309 Grm. Es wurde im $200 \mathrm{Mm}$.-Rohr eine Drehung von $95,4^{\circ}$ S.V.S. beobachtet, und berechnet sich hieraus eine Drehung von $196,764^{\circ} \alpha \mathrm{j}$.

55 Ccm. der Lösung gleich 5,119 Grm. Trockensubstanz, sind zu 250 Cem. gelöst und reducirten diese $0,48 \mathrm{Grm}$. Kupfer. 1 Grm. Trockensubstanz entspricht demnach 0,093 Grm. Kupfer. Diese 0,093 Grm. Kupfer entsprechen 0,0502 Traubenzucker. In $10 \mathrm{Grm}$. des Gemisches sind demnach 0,502 Grm. Traubenzucker und 9,498 Grm. Dextrin enthalten.

Die 0,502 Grm. Traubenzucker verlangen eine Drehung von $2,945^{\circ}(\alpha \mathrm{j})$.

9,498 Grm. Dextrin verlangen eine Drehung von $196,75^{\circ}$.

Die berechnete Gesammtdrehung beträgt $199,695^{\circ}$, statt der gefundenen $196,764^{0} \alpha$ j.

Versuch VII. 25 Grm. absolut reiner Stärke $(30,17$ lufttrocken.) sind mit 20 procent. Essigsäure 10 Stunden im Kochsalzbade in der Bombe erhitzt, alsdann zu $250 \mathrm{Ccm}$. aufgefüllt.

Das ermittelte spec. Gewicht betrug 1,0334. Daraus berechnet sich ein Trockengehalt von 9,226 Grm.

Die beobachtete Drehung betrug im $200 \mathrm{Mm} .-\mathrm{Rohr}$ $93^{\circ}$ S.V.S., die daraus berechnete spec. Drehung $\alpha \mathrm{j}=193,54^{0}$. $55 \mathrm{Ccm}$. dieser Lösung $=5,074 \mathrm{Grm}$. Trockensubstanz wurden 
332 Schulze: Die elementare Zusammensetzung

zu 250 Ccm. gelöst und reducirten 0,677 Grm. Kupfer. 1 Grm. Trockensubstanz entspricht demnach 0,133 Grm. Kupfer. Diese 0,133 Grm. Kupfer sind aber 0,0718 Grm. Traubenzucker entsprechend. In $10 \mathrm{Grm}$. des Gemisches sind demnach 0,718 Grm. Traubenzucker und 9,282 Grm. Dextrin enthalten.

Die $0,718 \mathrm{Grm}$. Traubenzucker verlangen eine Drehung von $4,213^{\circ}(\alpha \mathrm{j})$.

Die 9,282 Grm. Dextrin verlangen eine Drehung von $192,275^{0}(\alpha \mathrm{j})$.

Die berechnete Gesammtdrehung beträgt demnach $196,498^{\circ}$ statt der gefundenen $193,54^{\circ}(\alpha \mathrm{j})$.

Der besseren Uebersicht halber seien die in diesen vier Versuchen erhaltenen Resultate in einer Tabelle zusammengestellt: ${ }^{1}$ )

\begin{tabular}{c|c|c|c|c|c|c|c|}
\hline $\begin{array}{c}\text { Num. } \\
\text { des }\end{array}$ & $\begin{array}{c}\text { Zeit } \\
\text { der }\end{array}$ & $\begin{array}{c}\text { Gef. spec. } \\
\text { Gewicht. }\end{array}$ & $\begin{array}{c}\text { Berechnet. } \\
\text { Trocken- } \\
\text { gehalt. }\end{array}$ & $\begin{array}{c}\text { Gefund. } \\
\text { spec. } \\
\text { Drehg. } \alpha j\end{array}$ & $\begin{array}{c}\text { In } 10 \text { Grm. } \\
\text { enthalt. } \\
\text { Traubenz. }\end{array}$ & $\begin{array}{l}\text { Berechnet. } \\
\text { Gesarnmt- } \\
\text { Drehung. }\end{array}$ \\
\hline IV & 4 & 1,0304 & 8,398 & 199,361 & 0,426 & 200,83 \\
V & 6 & 1,0315 & 8,702 & 197,03 & 0,55 & 198,982 \\
VI & 8 & 1,0337 & 9,309 & 196,764 & 0,502 & 199,695 \\
VII & 10 & 1,0334 & 9,226 & 193,54 & 0,712 & 196,498
\end{tabular}

Aus diesen vier Versuchen ist ersichtlich, dass das Rotationsvermögen der Substanz mit der Zeit abnimmt und zwar in dem Maasse, als die Bildung des Traubenzuckers fortschreitet.

Der folgende Versuch, welcher mit einer grösseren Menge von Stärke ausgeführt wurde, zeigt die eben gemachte Wahrnehmung noch deutlicher.

Bei der Ausführung dieses Versuches wurden von Zeit zu Zeit Proben genommen, in denselben der Traubenzucker bestimmt, und so das Fortschreiten der Traubenzuckerbildung beobachtet.

1) Dass sich zwischen den gefundenen and berechneten spec. Drehungen Differenzen von ungefähr $3^{0}$ zeigen, führe ich darauf zurück, dass das Gemisch nicht aus reinem Dextrin $\alpha$ und Traubenzucker besteht, sondern dass in demselben noch andere Arten von Dextrin mit einer geringeren spec. Drehung enthalten sind. 
Versuch VIII. Hierbei sind $200 \mathrm{Grm}$. absolut reiner Stärke (241,42 Grm. lufttrockene) mit 20 proc. Essigsäure 14 Stunden am Rückflusskühler im Salzbade gekocht, nach dieser Zeit zu 2 Liter aufgefüllt und von dem ungelösten Rückstande abfiltrirt.

a) Gef. spec. Gewicht $=1,0375$, daraus berechneter Trockengehalt gleich 10,35 Grm.

Beobachtete Drehung im $200 \mathrm{Mm}$.Rohr $=100,6^{\circ}$ S.V.S.

Hieraus berechnete spec. Drehung $=186,458^{\circ}(\alpha \mathrm{j})$.

b) Der übrigbleibende Theil wurde gewogen und nochmals 6 Stunden am Rückflusskühler erhitzt, alsdann wieder zu dem ursprünglichen Gewichte aufgefüllt, und dann die Bestimmung des 'spec. Gewichts und der Polarisation ausgeführt.

Gef. spec. Gewicht $=1,0372$, daraus berechneter Trockengehalt $=10,276$ Grm.

Beobachtete Polarisation im $200 \mathrm{Mm} .-\mathrm{Rohr}=96,5^{0}$ S.V.S., berechnete spec. Drehung $\alpha \mathrm{j}=180,383^{\circ}$.

c) Die Lösung wurde ganz wie bei $b$. behandelt und weitere 8 Stunden gekocht.

Gef. spec. Gewicht $=1,0376$, berechneter Trockengehalt $=10,387 \mathrm{Grm}$.

Beobachtete Drehung im $200 \mathrm{Mm} .-\mathrm{Rohr}=96,5^{0} \mathrm{~S}$.V.S., berechnete spec. Drehung $\alpha \mathrm{j}=178,2805^{\circ}$.

d) Lösung wie bei $\mathrm{b}$ und $\mathrm{c}$ behandelt und nochmals 9 Stunden gekocht.

Gef. spec. Gewicht $=1,0372$, berechneter Trockengehalt gleich 10,276 Grm.

Beobachtete Drehung im $200 \mathrm{Mm} .-\mathrm{Rohr}=94^{\circ}$ S.V.S., berechnete spec. Drehung $=175,632^{\circ} \alpha \mathrm{j}$.

e) Lösung wie $b$ behandelt und nochmals 9 Stunden gekocht.

Gef. spec. Gewicht $=1,0375$, berechneter Trockengehalt gleich 10,359 Grm.

Beobachtete Drehung im $200 \mathrm{Mm}$-Rohr $=94^{0}$ S.V.S., berechnete spec. Drehung $=174,225^{\circ} \alpha \mathrm{j}$.

f) Der Rest der Lösung wurde nochmals 9 Stunden gekocht. 


\section{Schulze: Die elementare Zusammensetzung}

Gef. spec. Gewicht $=1,0375$, berechneter Trockengehalt gleich 10,359 Grm.

Beobachtete Drehung im $200 \mathrm{Mm}$.-Rohr $=90,8^{\circ}$ S.V.S., berechnete spec. Drehung. $=168,294^{0}$ aj.

\section{Berechnung des gebildeten Traubenzuckers und Dextrins.}

Bei Berechnung der Gesammtdrehung ist das von Bondonneau als Dextrin $\beta$ bezeichnete Dextrin mit einer spec. Drehung von $\alpha \mathrm{j}=196,099^{\circ}$ angewandt, weil die auf Dextrin $\alpha$ mit der spec. Drehung $\alpha \mathrm{j}=207,140^{\circ}$ berechneten Zahlen zu hoch ausfallen, während die auf Dextrin $\beta$ berechneteu mit den gefundenen annähernd übereinstimmen.

Ich führe hier nur die Resultate von a und $f$ an:

a) $50 \mathrm{Ccm}$. der Lösung $=5,179$ Grm. Trockensubstanz sind zu $250 \mathrm{Ccm}$. verdünnt und reducirten diese $0,375 \mathrm{Grm}$. Cu. $1 \mathrm{Grm}$. Trockensubstanz entspricht demnach 0,072 Grm. Cu. Diese 0,072 Grm. Cu sind gleich 0,0388 Grm. Traubenzucker. In $10 \mathrm{Grm}$. des Gemisches sind demnach 0,388 Grm. Traubenzucker und 9,612 Grm. Dextrin enthalten.

10 Grm. Trauberzucker bewirken cine Drehung von $58,68^{\circ} \alpha \mathrm{j}$, die $0,388 \mathrm{Grm}$. demnach eine solche von 2,276 $.10 \mathrm{Grm}$. Dextrin drehen $196,099^{\circ} \propto \mathrm{j} . \quad 9,612 \mathrm{Grm}$. demnach $188,49^{\circ} \propto \mathrm{j}$.

Die berechnete Gesammtdrehung beträgt demnach $190,766^{\circ} \propto j$, statt der gefundenen $186,458^{\circ} \propto j$. Die anf Dextrin $\alpha$ berechnete Gesammtdrehung beträgt $201,409^{\circ} \times \mathrm{j}$, statt der gefundenen $186,452^{\circ} \alpha \mathrm{j}$.

f) $50 \mathrm{Ccm}$. der Lösung, entsprechend 5,179 Grm. Trockensubstanz, sind zu $250 \mathrm{Ccm}$. aufgefüllt und reducirten diese 1,73 $\mathrm{Grm}$. Cu.

1 Grm. Trockensubstanz entspricht demnach 0,334 Grm. Cu., diese $0,334 \mathrm{Grm}$. Cu. sind aber 0,1803 Grm. Traubenzucker entsprechend.

In $10 \mathrm{Grm}$. des Gemisches sind demnach 1,803 Grm. Traubenzucker und 8,197 Grm. Dextrin enthalten. 10 Grm. Traubenzucker verlangen eine Drehung von $58,68^{\circ} \alpha \mathbf{j}, 1,803 \mathrm{Grm}$. demnach eine solche von $10,569^{\circ} \alpha$ j. 10 Grm. Dextrin $\beta$ verlangen eine Drchung von $196,099^{\circ} \alpha \mathrm{j}, 8,197$ Grm. demmach eine solche von $160,742^{\circ} \alpha \mathrm{j}$.

Die berechnete Gesammtdrehung beträgt $171,311^{\circ} \alpha \mathrm{j}$, statt der gefundenen $168,294^{\circ} \alpha j$. Die auf Dextrin $\alpha$ berechnete Gesammtdrehung beträgt $180,256^{\circ}$, statt der gefundenen $168,294^{\circ} \alpha \mathrm{j}$.

Der besseren Uebersicht wegen habe ich in der nachfolgenden Tabelle die verschiedenen bei Versuch VIII erhaltenen Zahlen zusammengestellt: 


\begin{tabular}{c|c|c|c|c|c|c}
\hline $\begin{array}{c}\text { Num. } \\
\text { der } \\
\text { Probe. }\end{array}$ & $\begin{array}{c}\text { Zeit } \\
\text { der }\end{array}$ & $\begin{array}{c}\text { Gechg. } \\
\text { Spec. } \\
\text { Gewicht. }\end{array}$ & $\begin{array}{c}\text { Berechn. } \\
\text { Trockeng. }\end{array}$ & $\begin{array}{c}\text { Gefund. } \\
\text { spec. } \\
\text { Drehung }\end{array}$ & $\begin{array}{c}\text { Berechn. } \\
\text { Gesammt- } \\
\text { drehung. }\end{array}$ & $\begin{array}{c}\text { In 10 Grm. } \\
\text { enthalt. } \\
\text { Traubenz. }\end{array}$ \\
\hline \hline a & $\mathbf{1 4}$ St. & 1,0375 & 10,359 & 186,458 & 190,766 & 0,388 \\
b & 20, & 1,0372 & 10,276 & 180,303 & & \\
c & 28, & 1,0376 & 10,387 & 178,280 & & \\
d & $37 "$ & 1,0372 & 10,276 & 175,632 & & \\
e & $46 "$, & 1,0375 & 10,359 & 174,225 & & \\
f & 55, & 1,0375 & 10,359 & 168,294 & 171,311 & 1,803
\end{tabular}

Wir ersehen aus diesen Zahlen ganz deutlich, dass mit abnehmendem Rotationsvermögen der Lösung der Gehalt derselben an Traubenzucker zunimmt.

Versuch IX. Dieser Versuch war bestimmt, den gebildeten Traubenzucker zu isoliren, und gebe ich daher lediglich nur die Operationen an, welche zu diesem Zwecke dienten und mit deren Hülfe es mir gelungen ist, den Traubenzucker möglichst rein darzustellen. $250 \mathrm{Grm}$. lufttrockener Stärke wurden mit soviel 20 procent. Essigsäure behandelt als erforderlich war, um am Schlusse der Reaction eine 10 procent. Lösung zu erhalten. Dieses Gemisch von Stärke und Essigsäure ist so lange am Rückflusskühler erhitzt, bis mit Jod keine Färbung und mit Alkohol keine Fällung mehr auftrat (hierzu waren 144 Stunden erforderlich); bis also anzunehmen war, dass die grösste Menge des zu Anfang gebildeten Dextrins in Traubenzucker umgewandelt war. Darauf wurde die Lösung von dem unlöslichen Rückstande abfiltrirt, abgedampft und so oft mit Wasser aufgenommmen und wieder abgedampft, bis sämmtliche Essigsäure verjagt war. Die stark gefärbte Lösung wurde nun mit gereinigter Knochenkohle behandelt und darauf bis zur schwachen Syrupconsistenz eingedampft.

Das spec. Gewicht dieser Lösung wurde za 1,1327 bestimmt und berechnet sich hieraus ein Trockengehalt von $34,829 \mathrm{Grm}$. (als Factor diente 3,81), welcher mit der directen Trockengehaltsbestimmung genau übereinstimmt.

Die Polarisation wurde im $100 \mathrm{Mm}$.-Rohr zu 75,750 S.V.S. gefunden, und berechnet sich hieraus eine spec. Drehung von $a j=83,516^{\circ}$. 


\section{Schulze: Die elementare Zusammensetzung}

Zur Bestimmung des gebildeten Traubenzuckers wurden $50 \mathrm{Ccm}$. der Lösung $=17,414$ Trockensubstanz zu $500 \mathrm{Ccm}$. verdünnt und von dieser Verdünnung wieder $100 \mathrm{Ccm} .=$ 3,482 Grm. zu $250 \mathrm{Ccm}$. verdünnt und reducirten diese 5,212 Grm. Cu. - 1 Grm. Trockensubstanz entspricht demnach 1,496 Grm. Cu. In 10 Grm. des Gemisches sind demnach 8,077 Grm. Traubenzucker und 1,923 Grm. Dextrin enthalten. $10 \mathrm{Grm}$. Traubenzucker verlangen eine Drehung von 58,68" $\alpha \mathrm{j}, 8,077 \mathrm{Grm}$. demnach eine solche von $47,395^{\circ} \propto \mathrm{j}$. $10 \mathrm{Grm}$. Dextrin $\beta$ drehen $196,099^{\circ} \alpha \mathrm{j}, 1,923 \mathrm{Grm}$. demnach $37,709^{\circ} \alpha \mathrm{j}$.

Die berechnete Gesammtdrehung beträgt demnach $85,104^{\circ} \alpha \mathrm{j}$, statt der gefundenen $83,516^{\circ} \alpha \mathrm{j}$. Auf Dextrin o berechnet beträgt die Gesammtdrehung $87,298^{\circ} \alpha \mathrm{j}$.

Die Substanz wurde nun mit Sand im Luftbade bei einer $90^{\circ}$ nicht übersteigenden Temperatur zur Trockene gedampft und dann mit Methylalkohol aufgenommen, um den Traubenzucker auszuziehen. Da jedoch von demselben alles aufgelöst wurde, so blieb nichts weiter übrig, als die Lösung zur starken Syrupconsistenz einzudampfen und sich selbst $\%$ überlassen.

Nach Verlauf von etwa 2 Monaten fing die Lösung an, an den Seiten des Gefässes auszukrystallisiren. Die Krystallisation wurde durch Mischen des Syrups mit den Krystallen wesentlich beschleunigt, so dass nach einigen Tagen die ganze Masse fest geworden war. Der Traubenzucker kounte jedoch in Folge des anhaftenden Dextrins nicht rein erhalten werden.

Dieses Gemisch wurde daher mit kochendem, absolutem Alkohol behandelt, durch welchen der Traubenzucker gelöst wurde, während das Dextrin ungelöst zurückblieb. Die Lösung wurde nun zur Syrupconsistènz eingedampft, und schied sich aus derselben der Traubenzucker in wohl ansgebildeten Krystallen ab.

Fin Theil der Krystalle mit dem Syrup wurde in Wasser gelöst und mit dieser Lösung das spec. Gewicht und die Polarisation bestimmt.

Gef. spec. Gew. $=1,04031$, daraus berechneter Trockengehalt $=10,58$ Grm. 
Beobachtete Drehung im $100 \mathrm{Mm}$.-Rohr $=16,1^{0}$ S.V.S. Daraus berechnet sich für $10 \mathrm{Grm}$. in $100 \mathrm{Ccm}$. gelöst eine spec. Drehung von $\alpha \mathrm{j}=58,435^{\circ}$, statt der von $10 \mathrm{Grm}$. Traubenzucker, in $100 \mathrm{Ccm}$. gelöst, verlangten $58,68 \mathrm{cj}$.

Es unterliegt demnach keinem $Z_{\text {weifel }}$ mehr, dass durch Einwirkung von verdünnter Essigsäure auf Stärkemehl Traubenzucker gebildet wird.

Zum Schluss seien die bei diesen Versuchen gemachten Beobachtungen hier übersichtlich zusammengestellt.

Behandelt man Stärke mit Essigsäure von mittlere Concentration und zwar zunächst vier Stunden lang unte $\mathbf{r}_{\mathbf{r}}$ Druck, so erhält man eine Lösung, welche mit Jod eine rothe Färbung und mit Alkohol einen weissen Niederschlag giebt; dieselbe reducirt Fehling'sche Lösung nur in sehr geringem Masse, besitzt aber ein ziemlich starkes Rotationsvermögen. Scheidet man nun den durch Alkohol fällbaren Körper ab, reinigt denselben auf die a. a. O. angegebene Weise und unterwirft denselben darauf einer genaueren Untersuchung, so findet man, dass derselbe ein Dextrin ist und zwar die von Bondonneau mit Dextrin $a$ bezeichnete Modification.

Nach meinen Beobachtungen besitzt das Dextrin $\propto$ eine spec. Drehung von $207,149^{\circ} a$ j und ein spec. Gewicht von 1,0362 (10 Grm. zu $100 \mathrm{Ccm}$. gelöst), reducirt aber Fehling'sche Lösung nicht und giebt mit Jod eine rothe Färbung.

Durch vierstündige Einwirkung der Essigsäure auf Stärkemehl wird dasselbe demnach fast ausschliesslich in Dextrin $\alpha$ verwandelt, welchem, wie schon oben gezeigt, Spuren von Traubenzucker beigemengt sind.

Wird nun die Einwirkung der Essigsäure fortgesetzt und durch von Zeit zu Zeit genommene Proben der weitere Verlauf der Reaktion beobachtet, so zeigt sich, dass das Rotationsvermögen der Lösung im Abnehmen, das Reductionsvermögen dagegen im steten Zunehmen begriffen ist. Diese beiden Erscheinungen stehen im innigsten Zusammenhange mit der immer mehr und mehr fortschreitenden Umwandlung Journal f. prakt. Chemie [2] Bd. 28 . 
338 Lerch: Ueber Brom- und Jodmagnesium.

des Dextrin $\alpha$ in Traubenzucker. Aus diesen Untersuchungen ergiebt sich somit, dass durch Behandeln der Stärke mit Essigsäure zunächst Dextrin und darauf Traubenzucker gebildet wird.

Braunschweig, chem.-techn. Laboratorium d. Herzogl. technischen Hochschule.

\section{Ueber Brom- and Jodmagnesium;}

\section{von \\ Otto Leroh.}

Die Verbindungen des Magnesiums mit Brom und Jod, sowie deren Hydrate und Doppelsalze, sind bisher Gegenstand eingehender Untersuchungen nicht gewesen.

So ist von dem wasserfreien Brommagnesium wenig mehr als dessen Existenz bekannt; das Jodmagnesium ist noch nicht dargestellt. Ueber die Hydrate und Doppelsalze beider Körper finden sich nur vereinzelte Angaben.

Der Verfasser hat sich bestrebt, diese Lücke auszufüllen. Es ist ihm gelungen, das wasserfreie Bromid, auch das Jodid darzustellen, desgleichen die Hydrate und einige Doppelsalze.

\section{Brommagnesium.}

Die ältesten Angaben über diesen Körper rühren von Serullas ${ }^{1}$ ) (1832) her. Er bemerkte nämlich, dass beim Ueberleiten von Bromdampf über ein glühendes Gemenge von Kohle und Magnesia eine geschmolzene Masse entstand, die er für Brommagnesium hielt. Er beobachtete, dass ein Theil dieser Substanz in die Vorlage übergehe, und dass ein anderer Theil im Rohre zurückbleibe. Er beschreibt dieselbe als nicht flüchtig, in der Rothgluth schmelzend und von krystallinischem Gefüge. Denselben Weg der Darstellung beschritt Löwig und fügte den Beobachtungen Serullas'

1) Pogg. Ann. 24, 343. - Journ. chim. méd. 8, 4. 